\title{
Do stroke patients with or without cardiac intervention achieve similar functional recovery? - functional recovery and rehabilitation efficiency of complex stroke patients following cardiac intervention
}

\begin{abstract}
A complex stroke (stroke occurring in the context of a cardiac intervention) is a well recognised entity but not well researched in terms of functional outcomes and efficiency of rehabilitation. The implication for prognosis and rehabilitation services is uncertain. We describe the functional outcomes of complex stroke patients following rehabilitation compared with stroke patients in the absence of cardiac intervention. Total 94 ischemic stroke patients were included (70 non-complex and 24 complex strokes). Measures of function, rate of recovery, efficiency of rehabilitation and markers of outcome were analysed. There were no significant differences in length of stay between two cohorts. A non-significant trend towards better functional profiles on discharge and greater improvement from baseline was observed in the complex strokes as compared with the non-cardiac intervention group.
\end{abstract}

Keywords: complex stroke, cardiac intervention, rehabilitation, functional outcomes, fim, functional independent measures
Volume 2 Issue I - 2018

\author{
Ling, Lan, 'Tarinisha Shama, ${ }^{2}$ Leah Thompson, ${ }^{3}$ \\ MoCcThySt ${ }^{2}$ \\ 'Geriatric and Rehabilitation Medicine, The Prince Charles \\ Hospital, Australia \\ ${ }^{2}$ Community Based Rehabilitation Team, MNHHS, Australia \\ Internal Medicine Services, The Prince Charles Hospital, \\ Australia
}

Correspondence: Ling Lan, Geriatric and Rehabilitation Medicine, The Prince Charles Hospital, Rode Road, Chermside, Queensland,Australia, Email ling.lan@health.qld.gov.au

Received: December 21, 2017 | Published: February 02, 2018

\section{Introduction}

The epidemiology of stroke in patients undergoing cardiac intervention has been previously documented. ${ }^{1}$ The reported incidence of stroke after cardiac surgery varies depending on the procedure and whether the findings have been obtained prospectively or retrospectively. The stroke incidence with coronary artery bypass grafting has been reported from $1.5 \%$ to $5.2 \%$ in prospective studies as compared with $0.8 \%$ to $3.2 \%$ in retrospective studies. ${ }^{2-5}$ Incidence of stroke can be as high as $9.7 \%$ following valvular surgery. ${ }^{6}$ One of the most serious adverse complications associated with percutaneous coronary interventions is stroke, reported to occur in $0.07 \%$ to $0.3 \%$ of all percutaneous coronary procedures. ${ }^{6-10}$ Transcatheter aortic valve implantation has been used increasingly to treat symptomatic patients with severe aortic stenosis. Although mortality rates have been declining with increasing operator experience and device improvements, cerebrovascular complications continue to occur. ${ }^{11-13}$

Addressing cerebrovascular complications after cardiac interventions is important for several reasons. Strokes occurring immediately after surgical interventions have devastating consequences; apart from high mortality rates, ${ }^{3}$ the supposed benefits of such interventions in improving quality of life in these patients may be abolished. Equally, the cost-effectiveness of interventions such as transcatheter aortic valve implantation undertaken in high risk patients may also be lost. While technical innovations have allowed correction of many acquired structural cardiac anomalies in high risk and elderly patents, their rehabilitation and functional outcomes are poorly defined and remain a highly debated issue.

Although few studies have reviewed the short and long-term outcomes of stroke after cardiac surgery, most available studies are targeted on survival rate, mortality rate and predicting risk factors. ${ }^{14-16}$ There is a significant paucity of data pertaining to functional outcomes of complex strokes that occur post cardiac interventions. One study on the post-cardiac surgery stroke patient focused on the cognitive assessment and its impact on the participation of rehabilitation. ${ }^{17}$ Given the lack of knowledge about whether the complex stroke patient post cardiac intervention achieves similar functional recovery, an observational cohort study was conducted. The study aimed to provide further preliminary insights into the role of rehabilitation and functional outcomes in patients with a dual diagnosis of complex stroke by using well defined internationally standardised measures including functional independent measures (FIM), efficiency of rehabilitation, discharge destinations and level of community support.

\section{Methods}

A retrospective consecutive chart review was performed after obtaining local ethics approval (HREC/13/QTPCH/37) at The Prince Charles Hospital, which is a leading tertiary cardio-thoracic hospital in Australia. Patients who were admitted to the Rehabilitation and Acute Stroke unit in The Prince Charles Hospital following an ischemic stroke with or without cardiac intervention between September 2011 and 2013 were included in this observational cohort study. Patients were excluded from this study if they had haemorrhagic stroke or were unable to participate in rehabilitation due to either severe cognitive impairment or uncontrolled psychiatric conditions. Complex stroke was defined as stroke occurring within 30 days of cardiac intervention. Non-complex stroke was defined as a clinical ischemic stroke occurring without cardiac intervention. All diagnosis of ischemic stroke was confirmed by both clinical and medical imaging studies of either Computerized Tomography or Magnetic Resonance Imaging of brain as appropriate. 
Data pertaining to patient demographics, cardiac intervention performed, functional profiles including FIM, Length of Stay (LOS), FIM efficiency (FIM gain divided by LOS), and episodes of complications requiring transferring back to acute care, discharge destination, and level of care support on admission and upon discharge from rehabilitation unit were collected. Discrete variables were expressed as counts (percentage) and continuous variables as Median (interquartile range) \pm Standard Error (SE). Statistical analyses were performed using two sampled student $t$-tests, in addition to the use of descriptive statistics. A $p<0.05$ was considered to be statistically significant.

\section{Results}

\section{Demography and general outcome measures}

Total 126 stroke patients were admitted to the rehabilitation unit during 2011 and 2013. Ninety four (94) patients with ischemic stroke were included in this study. Seventy (70) were identified to have non- complex strokes and 24 sustained complex strokes. The proportion of stroke post cardiac intervention in the rehabilitation setting was $19 \%$ of total stroke admissions. Median age of patients was $69 \pm 2.13$ years for patients with complex strokes and $72 \pm 2.28$ for non-complex strokes $(\mathrm{p}=0.78)$. The baseline demographic data is presented in Table 1 . Both study cohorts had similar significant co-morbidities which are considered risk factors for stroke (hypertension, diabetes, dyslipidemia, and vascular disease).

\section{Functional outcomes and rehabilitation efficiency}

Rehabilitation outcomes at admission and discharge are presented in Table 2. Functional profiles (FIM motor, FIM cognition and FIM total) on admission of complex patients were non-significantly lower than non-complex patients. Functional profiles on discharge of complex patients were slightly higher than non-complex patients but there was no statistically significant difference. There were no significant differences in FIM efficiencies between non-complex and complex stroke patients (Table 2).

Table I The baseline characteristics of the study population

\begin{tabular}{|c|c|c|c|}
\hline Characteristics & Complex stroke $n=24$ & Non-complex stroke $n=70$ & PValue \\
\hline Age, Median ( \pm SE ) & $69( \pm 2.13)$ & $72( \pm 2.28)$ & 0.78 \\
\hline \multicolumn{4}{|l|}{ Sex, n(\%) } \\
\hline Male & 17(7|\%) & $44(63 \%)$ & 0.8 \\
\hline Female & $7(29 \%)$ & $26(37 \%)$ & 0.65 \\
\hline Language (English), n(\%) & $24(100 \%)$ & $68(97 \%)$ & 0.41 \\
\hline Ethnic minority, n(\%) & $2(8 \%)$ & $9(13 \%)$ & 0.45 \\
\hline No. of comorbidities, median $( \pm S E)$ & $5( \pm 0.18)$ & $5( \pm 0.12)$ & 0.49 \\
\hline Home dwelling, n(\%) & $24(100 \%)$ & $66(94 \%)$ & 0.24 \\
\hline Carer, n(\%) & $3(13 \%)$ & $13(18 \%)$ & 0.7 \\
\hline Domestic services, $n(\%)$ & $\mathrm{I}(4 \%)$ & $8(11 \%)$ & 0.3 \\
\hline \multirow[t]{4}{*}{$\begin{array}{l}\text { Independent ambulation, } \mathrm{n}(\%) \\
\text { (with or without aids) }\end{array}$} & $24(100 \%)$ & $68(97 \%)$ & 0.11 \\
\hline & CABG - 8 & & \\
\hline & AVR - 4 & & \\
\hline & MVR - 2 & & \\
\hline \multirow[t]{4}{*}{ Cardiac interventions, $\mathrm{n}$} & $A V R+M V R-4$ & $\mathrm{~N} / \mathrm{A}$ & N/A \\
\hline & Arch repair - 2 & & \\
\hline & PFO closure - I & & \\
\hline & AVR/CABG/root repair) - 3 & & \\
\hline
\end{tabular}

N, number of cases, Ethnic Minority, Non-Caucasian Descent; Comorbidities including risk factors for ischemic stroke such as hypertension; Dyslipidemia, smoking, ischemic heart disease; atrial fibrillation;Type 2 diabetes mellitus; peripheral artery disease, obstructive sleep apnoea, etc. Independent ambulation aids, ability to walk with or without assistive device including a single point stick or a wheeled walker; Cardiac interventions involving sternotomy surgery; CABG, coronary artery bypass grafting;AVR, aortic valve repair; MVR, mitral valve repair; PFO, patent foramen ovale; N/A, nil/applicable. 
Table 2 Functional outcomes (total FIM scores at admission and discharge, FIM efficiency, and length of stay) and discharge destinations between non-complex and complex stroke patients

\begin{tabular}{llllll}
\hline Median ( \pm SE ) & aFIM & dFIM & FIM Gains & FIM Efficiency & LOS \\
\hline Non-complex & $88( \pm 3.38)$ & $112( \pm 3.97)$ & $24( \pm 1.65)$ & $0.96( \pm 0.15)$ & $25( \pm 3.45)$ \\
Complex & $7 I( \pm 6.56)$ & $113( \pm 3.33)$ & $42( \pm 4.83)$ & $1.35( \pm 0.17)$ & $31( \pm 4.65)$ \\
P value & 0.65 & 0.23 & 0.21 & 0.85 & 0.77
\end{tabular}

aFIM, total fim scores at admission; dFIM, total fim scores at discharge; LOS, length of stay; FIM gains, FIM scores at discharge minus FIM scores at admission; FIM efficiency, FIM gains divided by LOS

Functional outcomes and rehabilitation efficiency

\begin{tabular}{llll}
\hline Measures & Non-complex $\mathbf{n = 7 0}$ & Complex $\mathbf{n = 2 4}$ & PValue \\
\hline Discharge to home rate (\%) & $60(86 \%)$ & $22(92 \%)$ & 0.45 \\
Discharge support rate (\%) & $38(63 \%)$ & $8(36 \%)$ & 0.06
\end{tabular}

Rehabilitation outcomes in discharge destination.

\section{Rehabilitation outcomes}

Although, complex stroke patients experienced more cardiac related complications such as exacerbation of heart failure and fasting atrial fibrillation requiring more investigations and acute medical interventions, there was no significant differences in hospital length of stay (Table 2) between patients who sustained complex and noncomplex strokes $(31 \pm 4.65$ vs. $25 \pm 7.54$ days, $p=0.77)$. Total 10 out of 70 non-complex patients were discharged to a nursing home compared to 1 out of 24 complex patients. One of 24 patients with complex stroke was transferred to another hospital. Sixty (60) non-complex patients returned to home, of which 38 were discharged home with community support as compared to 8 out of 22 complex patients who returned home (Table 2). Patients with non-complex stroke required more community support upon discharge $(63 \%)$ than complex patients $(36 \%, p=0.06)$. Patients with complex stroke seemed to have more favourable outcome in discharge destination than the cohort with noncomplex stroke.

\section{Discussion}

This preliminary study provides useful insights into functional rehabilitation outcomes following complex strokes sustained in the setting of cardiac intervention. This study compares and contrasts the basic demographic and functional outcome differences between the two cohorts. The proportion of complex stroke in The Prince Charles Hospital Rehabilitation and Acute Stroke unit is higher than the literature, which may reflect the complex diagnostic caseload of cardio thoracic patients in this tertiary hospital, which is one of the leading cardio-thoracic hospitals in Australia. Interestingly, the percentage of patient's discharged home and level of support upon discharge did not differ significantly between the two groups. Equally, the functional measures upon admission and discharge from the unit were not significantly different, though the functional profile of complex patients were moderately lower than their counterpart at admission.

The findings of rehabilitation outcomes indicate that complex strokes may have a comparable outcome to non-complex stroke patients. Patients with complex strokes were slightly younger and more mobile. It is likely that the patients who were physically and medically stable due to young age were offered cardiac interventions as compared with more frail patients (for example nursing home residents) included in non-complex stroke cohort. It should be emphasised that such age distinction is likely to diminish over time with more cardiac interventions such as transcatheter aortic valve implantation now being performed in elderly patients. ${ }^{18}$

Surprisingly, despite a lower age, patients with complex strokes recorded lower functional independence measures upon admission to the Rehabilitation and Acute Stroke unit. This may be due to a greater severity of stroke and post intervention complications. However, it should also be noted that significant pathophysiological differences exist between complex and non-complex strokes and many of which may not be quantified. The FIM measures at discharge were generally higher in complex patients than patients with non- complex strokes, reflective of greater age and frailty in the later cohort. FIM efficiency was regained at a much faster rate in complex patients for similar reasons such as improved cardiac function post intervention and subsequent improvement in the cerebral perfusion.

It is likely that age and co-morbidities play a predominant role in stroke outcome regardless of complexity of stroke. Rehabilitation physicians should carefully balance these demographic and clinical factors along with severity of stroke prior to exploring the rehabilitation potential of such patients. The changing demography of complex strokes in the modern era even makes a case for more proactive involvement of rehabilitation physicians in the preoperative assessment of patients undergoing cardiac interventions. The complexity of the stroke in itself may not be a true reflection of rehabilitation potential as observed in this study.

This preliminary study has limitations, most importantly the sample size and its retrospective nature. We acknowledge the limitation of imbalanced quantity of complex stroke patients compared to noncomplex patients, may cause analysis bias. This imbalance increases the likelihood of false negative findings of non-significant difference between two groups. However, we believe that the data represents a unique dataset that could still have relevance to other stroke centres, as it represents an enriched sample of complex stroke patients due to the high volume of cardiac surgeries undertaken in high risk patients within a tertiary cardiac centre. The study cohort of complex stroke is relatively young and complex strokes in the elderly may have different outcomes. This calls for well-designed, large prospective studies which include rehabilitation and long term functional outcomes rather than focussing on survival alone. 


\section{Conclusion}

Patients with complex stroke following cardiac intervention compared with non-complex strokes had similar functional rehabilitation outcomes. With an ageing population, the elderly and frail are more likely to undergo cardiac interventions and suffer complex strokes in future. The functional outcomes from complex stroke patients may dictate the viability of such interventions. Complex strokes are an emerging population in the rehabilitation setting and further prospective studies are warranted.

\section{Acknowledgements}

We would like to thank the patients and the staff of the Rehabilitation Day Therapy Unit at the Prince Charles Hospital in Queensland.

\section{Conflict of interest}

The author declares no conflict of interest.

\section{References}

1. Anyanwu AC, Filsoufi F, Salzberg SP, et al. Epidemiology of stroke after cardiac surgery in the current era. $J$ Thorac Cardiovasc Surg. 2007;134(5):1121-1127.

2. Breuer AC, Furlan AJ, Hanson MR, et al. Central nervous system complications of coronary artery bypass graft surgery: Prospective analysis of 421 patients. Stroke. 1983;14(5):682-687.

3. McKhann GM, Goldsborough MA, Borowicz LM, et al. Predictors of stroke risk in coronary artery bypass patients. Ann Thorac surg. 1997;63(2):516-521.

4. Martin WR, Hashimoto SA. Stroke in coronary bypass surgery. The Canadian journal of neurological sciences. Le Journal Canadien Des Sciences Neurologiques. 1982;9(1):21-26.

5. Coffey CE, Massey EW, Roberts KB, et al. Natural history of cerebral complications of coronary artery bypass graft surgery. Neurology. 1983;33(11):1416-1421.

6. McKhann GM, Grega MA, Borowicz LM, et al. Stroke and encephalopathy after cardiac surgery: An update. Stroke. 2006;37(2):562-571.

7. Weintraub WS, Mahoney EM, Ghazzal ZM, et al. Trends in outcome and costs of coronary intervention in the 1990s. Am J Cardiol. 2011;88(5):497-503.
8. Batchelor WB, Anstrom KJ, Muhlbaier LH, et al. Contemporary outcome trends in the elderly undergoing percutaneous coronary interventions: Results in 7,472 octogenarians. National cardiovascular network collaboration. J Am Coll Cardiol. 2000;36(3):723-730.

9. Cubo E, Estefania CM, Monaco M, et al. Risk factors of stroke after percutaneous transluminal coronary angioplasty. Eur J Neurol. 1998;5(5):459-462.

10. Fuchs S, Stabile E, Kinnaird TD, et al. Stroke complicating percutaneous coronary interventions: Incidence, predictors, and prognostic implications. Circulation. 2002;106(1):86-91.

11. Webb JG, Altwegg L, Boone RH, et al. Transcatheter aortic valve implantation: Impact on clinical and valve-related outcomes. Circulation. 2009;119(23):3009-3016.

12. Himbert D, Descoutures F, Al-Attar N, et al. Results of transfemoral or transapical aortic valve implantation following a uniform assessment in high-risk patients with aortic stenosis. J Am Coll Cardiol. 2009;54(4): 303-311.

13. Webb JG, Pasupati S, Humphries K, et al. Percutaneous transarterial aortic valve replacement in selected high-risk patients with aortic stenosis. Circulation. 2007;116(7):755-763.

14. Salazar JD, Wityk RJ, Grego MA, et al. Stroke after cardiac surgery: short- and long-term outcomes. Ann Thorac Surg. 2001;72(4):11951201 .

15. Guzman LA, Costa MA, Angiolillo DJ, et al. A systemic review of outcomes in patients with staged carotid artery stenting and coronary artery bypass graft surgery. Stroke. 2008;39(2):361-365.

16. Domanski MJ, Farkouh ME, Zak V, et al. Predictors of stroke associated with coronary artery bypaass grafting inpatients with diabetes mellitus and multivessel coronary artery disease. Am J cardiol. 2015;115(10):13821388 .

17. Oden KE, Kevorkian CG, Levy JK. Rehabilitation of the post-cardiac surgery stroke patient: Analysis of cognitive and fucntional assessment. Arch Phys Med Rehabil. 1998;79(1):67-71.

18. Fanning JP, Walters DL, Eeles E, et al. Characterization of neurological injury in transcatherter aortic valve implantation How clerr is the picture? Circulation. 2014;129(4):504-515. 